\title{
Specific peripheral B cell tolerance defects in patients with multiple sclerosis
}

Tuure Kinnunen, ${ }^{1}$ Nicolas Chamberlain, ${ }^{2}$ Henner Morbach, ${ }^{2}$ Tineke Cantaert, ${ }^{2}$ Megan Lynch, ${ }^{2}$ Paula Preston-Hurlburt, ${ }^{2}$ Kevan C. Herold, ${ }^{2,3}$ David A. Hafler, ${ }^{1}$ Kevin C. O'Connor, ${ }^{1}$ and Eric Meffre ${ }^{2}$

1Department of Neurology, ${ }^{2}$ Department of Immunobiology, and ${ }^{3}$ Department of Internal Medicine, Section of Endocrinology and Metabolism, Yale University School of Medicine, New Haven, Connecticut, USA.

\begin{abstract}
Multiple sclerosis (MS) is a genetically mediated autoimmune disease of the central nervous system. B cells have recently emerged as major contributors to disease pathogenesis, but the mechanisms responsible for the loss of B cell tolerance in patients with MS are largely unknown. In healthy individuals, developing autoreactive $B$ cells are removed from the repertoire at 2 tolerance checkpoints during early $B$ cell development. Both of these central and peripheral $B$ cell tolerance checkpoints are defective in patients with rheumatoid arthritis (RA) and type 1 diabetes (T1D). Here, we found that only the peripheral, but not the central, B cell tolerance checkpoint is defective in patients with MS. We show that this specific defect is accompanied by increased activation and homeostatic proliferation of mature naive B cells. Interestingly, all of these MS features parallel defects observed in FOXP3-deficient IPEX patients, who harbor nonfunctional Tregs. We demonstrate that in contrast to patients with RA or T1D, bone marrow central B cell selection in MS appears normal in most patients. In contrast, patients with MS suffer from a specific peripheral B cell tolerance defect that is potentially attributable to impaired Treg function and that leads to the accumulation of autoreactive $B$ cell clones in their blood.
\end{abstract}

\section{Introduction}

Multiple sclerosis (MS) is an inflammatory disease of the central nervous system (CNS) caused by an autoimmune response in genetically susceptible individuals (1). Although $\mathrm{T}$ cells are considered to be the main effectors in MS pathogenesis, recent studies have highlighted the important contribution of B cells to disease progression (2). Perhaps the strongest evidence for a major role of $\mathrm{B}$ cells in MS is the substantial clinical benefit achieved with antiCD20 (rituximab) therapy-mediated peripheral B cell depletion (3). However, the underlying mechanisms by which B cells contribute to autoimmunity in MS remain largely unknown.

We have previously established that most developing autoreactive B cells in healthy individuals are removed at 2 discrete steps. A central B cell tolerance checkpoint in the bone marrow removes the vast majority of $B$ cell clones expressing polyreactive antibodies (4). A peripheral B cell tolerance checkpoint further counterselects autoreactive new emigrant $B$ cells before they enter the mature naive B cell compartment (4). Both central and peripheral B cell tolerance checkpoints are defective in untreated patients with active rheumatoid arthritis (RA) or type 1 diabetes (T1D) $(5,6)$. As in MS, anti-CD20 therapy has also shown some efficacy in the treatment of RA and to a lesser extent T1D, suggesting that the deletion of autoreactive B cell clones may either prevent or delay autoimmune manifestations $(7,8)$.

\section{Results and Discussion}

To determine whether $\mathrm{B}$ cell tolerance checkpoints are defective in MS patients, we tested the reactivity of recombinant antibodies cloned from 181 single new emigrant/transitional and 169 mature naive B cells (sorting strategy shown in Supplemental Figure 1; supplemental material available online with this article; doi:10.1172/

Conflict of interest: The authors have declared that no conflict of interest exists. Citation for this article: J Clin Invest. 2013;123(6):2737-2741. doi:10.1172/JCI68775.
JCI68775DS1) from 7 treatment-naive MS patients (Supplemental Table 1) and compared it with that of recombinant antibodies cloned from their B cell counterparts in 11 healthy donors (refs. 4, 9-11, and Supplemental Table 1) and in patients with T1D or RA $(5,6)$. We found that the proportion of new emigrant/transitional polyreactive $\mathrm{B}$ cells in MS was low and comparable to that in healthy donors in a large majority of the patients (5 of 7) and was elevated in only 2 MS patients (MS01 and MS04, 32.1\% and 19.4\%, respectively) (Figure 1, A and B, Supplemental Tables 2-8, and ref. 4). The frequency of antinuclear new emigrant/transitional B cells in MS patients was also comparable to that in healthy donors (Figure 1C). These findings demonstrate that central B cell tolerance is normally established in the majority of MS patients. In contrast, all RA and T1D patients display a defective central B cell tolerance checkpoint as illustrated by the high frequencies of polyreactive new emigrant/transitional $\mathrm{B}$ cells in these patients (Figure $1 \mathrm{~B}$ and refs. 5, 6). We conclude that defects in central B cell tolerance are not found in most patients with MS, whereas they are a common feature of RA and T1D.

Autoreactive B cell clones that recognize peripheral self-antigens may be further eliminated at a second B cell tolerance checkpoint before they enter the mature naive B cell compartment (4). To determine whether this peripheral B cell tolerance checkpoint is functional in MS, we tested the reactivity of recombinant antibodies from mature naive B cells from MS patients against HEp- 2 cell lysates by ELISA (Figure 2, A and B). We found that the frequency of mature naive B cells expressing HEp-2-reactive antibodies was significantly increased in all 7 MS patients $(33.3 \%-61.1 \%)$ compared with the healthy donors $(16.0 \%-26.3 \%, P<0.001)$ (Figure 2 , A and B, and Supplemental Tables 9-15). The high proportion of HEp-2-reactive clones in the mature naive B cell compartment in MS patients was similar to that in T1D and RA patients (Figure 2B and refs. 5, 6). Peripheral B cell tolerance checkpoint defects in MS patients were further evidenced by the increased frequency of mature naive B cells expressing polyreactive antibodies in these patients compared with 
A New emigrant/transitional B cells
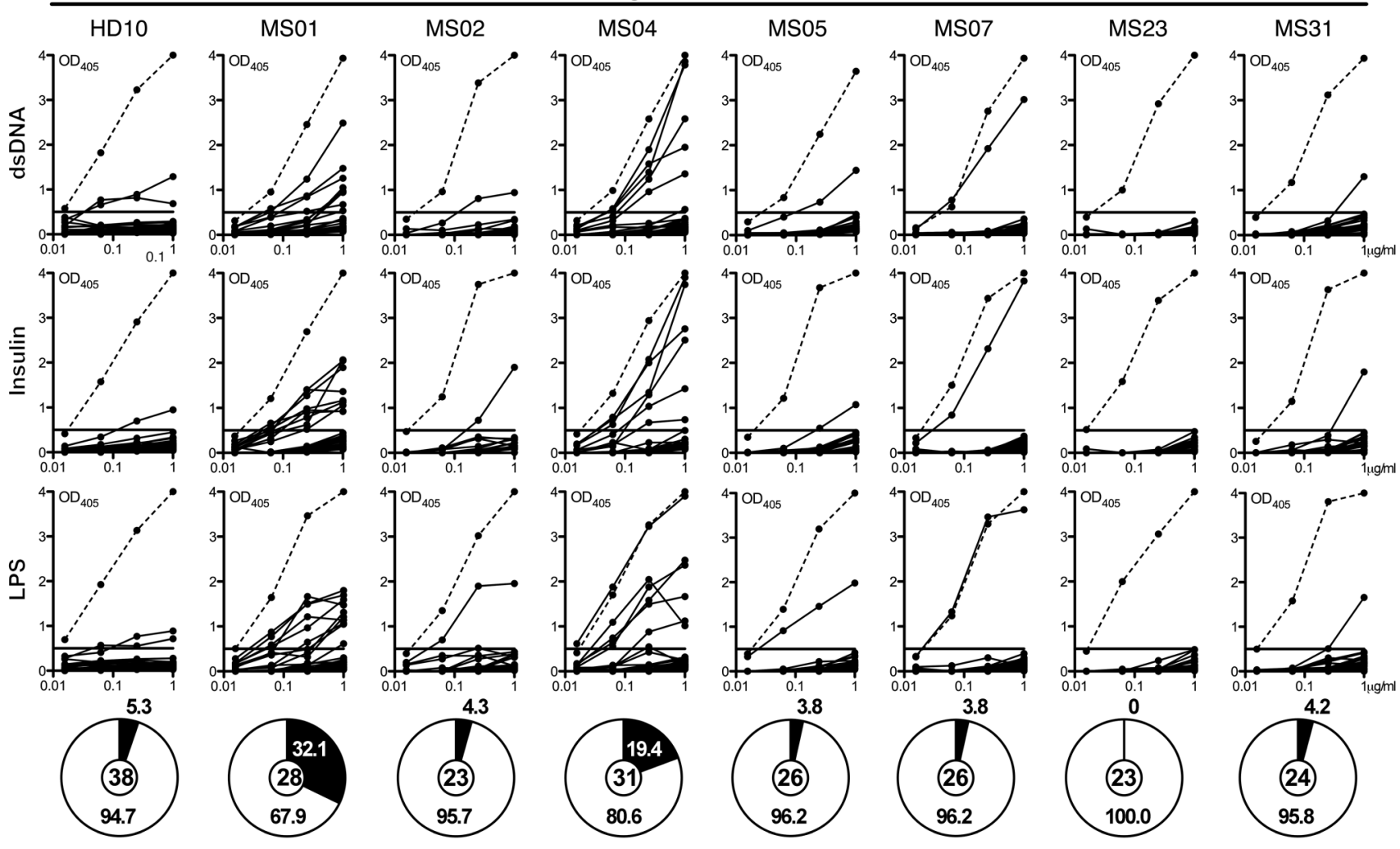

B

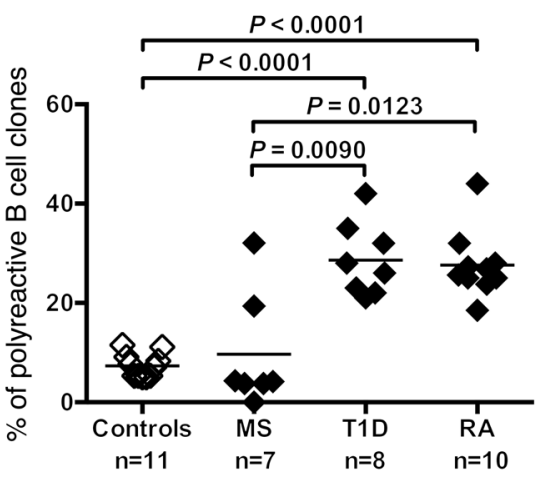

C

Nonpolyreactive (\%)

Polyreactive (\%)

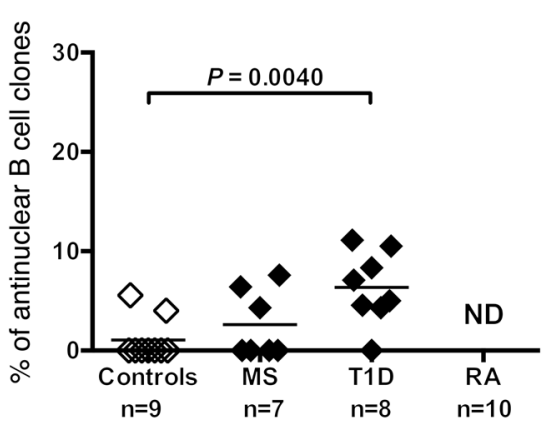

Figure 1

Central B cell tolerance is functional in most MS patients. (A) Antibodies from new emigrant/transitional B cells from a healthy donor and MS patients were tested by ELISA for reactivity against dsDNA, insulin, and LPS. Dotted lines show the ED38-positive control, and solid lines show binding for each cloned recombinant antibody. Horizontal lines define the cutoff $\mathrm{OD}_{405 \mathrm{~nm}}$ for positive reactivity. Frequency of polyreactive (filled area) clones is summarized in the pie charts, with the total number of clones tested indicated in the centers. Frequencies of polyreactive (B) and antinuclear (C) new emigrant/transitional B cells in MS patients are similar to those in healthy controls and differ from those in T1D and RA patients. Statistical differences are indicated when significant.

healthy controls $(P=0.0076)$ (Figure 2C and Supplemental Figure 2). Polyreactive recombinant antibodies expressed by mature naive $\mathrm{B}$ cells from MS patients also reacted against a protein extract derived from brain white matter, which contains CNS antigens such as MBP and MOG (Figure 2D). However, in contrast to T1D patients, there was no increase in antinuclear clones in mature naive $\mathrm{B}$ cells from MS patients (Figure 2E). Thus, MS patients suffer from an impaired peripheral B cell tolerance checkpoint, resulting in the accumulation of large numbers of autoreactive and polyreactive mature naive B cells, which can express white matter-reactive antibodies.
Our recent findings demonstrate that Tregs play an important role in the establishment of peripheral B cell tolerance. Indeed, IPEX patients who suffer from impaired Treg functions due to mutations in the FOXP3 gene display specific defects in the peripheral B cell tolerance checkpoint associated with altered B and T cell phenotype and homeostasis (12). To investigate whether the defective peripheral B cell tolerance in MS patients recapitulates features observed in IPEX patients, we first analyzed the phenotype and the in vivo replication history of B cells from MS, T1D, and RA patients. Reminiscent of B cells from IPEX patients, naive B cells 
A Mature naive $B$ cells

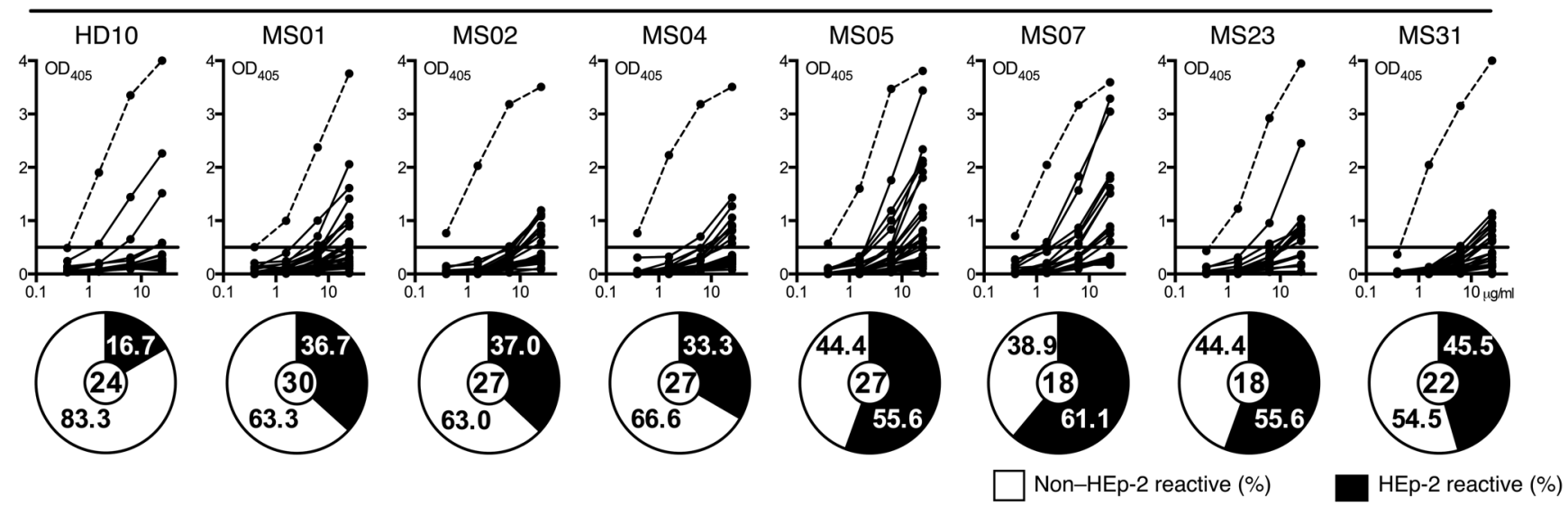

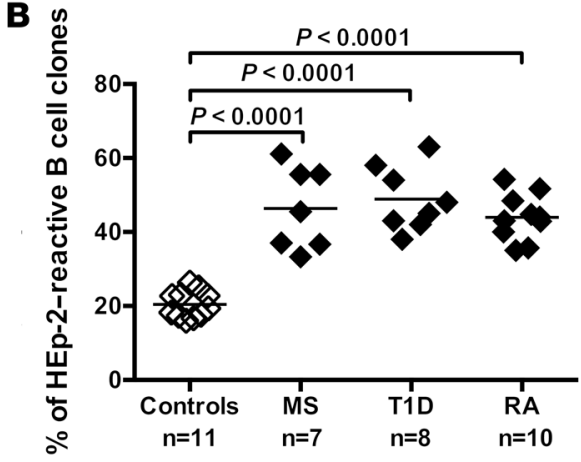

D

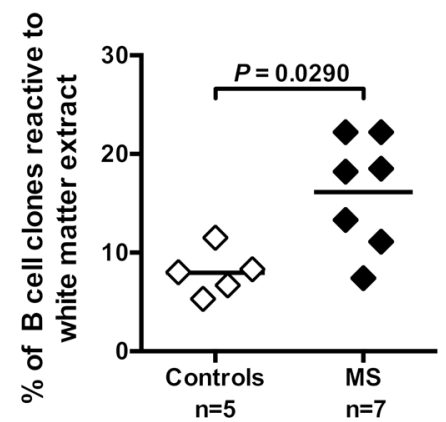

C

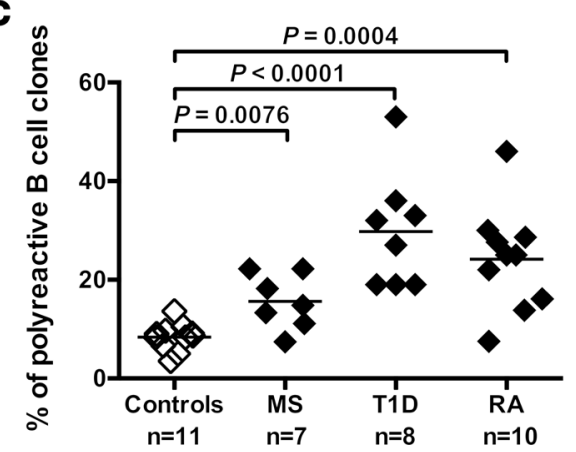

E

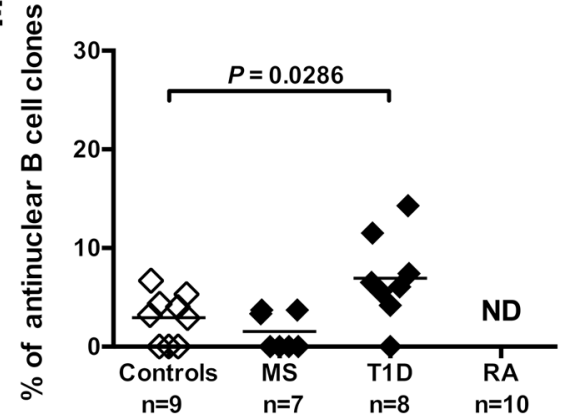

Figure 2

Peripheral $B$ cell tolerance is defective in MS patients. (A) Antibodies from mature naive $B$ cells from a healthy donor and MS patients were tested by ELISA for anti-HEp-2 cell reactivity. Dotted lines show the ED38-positive control, and solid lines show binding for each cloned recombinant antibody. Horizontal lines define the cutoff $\mathrm{OD}_{405 \mathrm{~nm}}$ for positive reactivity. Frequency of HEp-2-reactive (filled area) clones is summarized in the pie charts, with the total number of clones tested indicated in the centers. Frequencies of HEp-2-reactive (B), polyreactive (C), and white matter extract-reactive (D), but not antinuclear (E), mature naive B cells are increased in MS patients compared with healthy controls. Statistical differences are indicated when significant. from MS and T1D patients displayed a modestly activated phenotype characterized by a lack of CD80 and CD86, but a significantly upregulated expression of the CD69 activation marker, which supports the recruitment of B cells to lymph nodes (Figure 3, A and B, Supplemental Figure 3, and ref. 12). We assessed the proliferative history of new emigrant/transitional and mature naive B cells from patients and controls by measuring $\kappa$-deleting recombination excision circles (KRECs), which provide an estimate of the number of divisions a $\mathrm{B}$ cell population has undergone (13). We found that mature naive B cells from MS, T1D, and RA patients displayed increased homeostatic proliferation, similar to that observed in IPEX patients, with an average of 2.6 to 2.8 divisions in vivo compared with 2.0 divisions in B cells from healthy donors (Figure 3C, right, and ref. 12). An increased proliferative history was restricted to the mature naive compartment, whereas the proliferative history of new emigrant/transitional B cells from these patients was similar to that of healthy controls (Figure 3C, left). The increased B cell proliferation in autoimmune patients and their defective periph- eral B cell tolerance checkpoint was not due to increased concentrations of serum $B$ cell activating factor (BAFF), which is a critical $B$ cell survival factor that controls the number of peripheral B cells (14), because serum BAFF levels were comparable between healthy individuals and patients with MS and T1D but were increased in untreated RA patients (Figure 3D). We conclude that B cells from MS, T1D, and RA patients display an altered phenotype and proliferative history similar to what has been previously observed in IPEX patients with defective Treg function (12).

To further evaluate the contribution of Treg dysfunction to B cell tolerance defects in autoimmunity, we assessed the phenotype of Tregs from autoimmune disease patients. We observed that the proportion of $\mathrm{CD}_{45 \mathrm{RO}^{+}}$memory Tregs was increased in untreated MS patients compared with age-matched healthy controls (Supplemental Figure 4 and refs. 15, 16). A similar phenomenon was found in RA, but not in T1D patients (Supplemental Figure 4). Interestingly, the defective peripheral B cell tolerance checkpoint observed in IPEX patients also correlates with a Treg compartment 
A

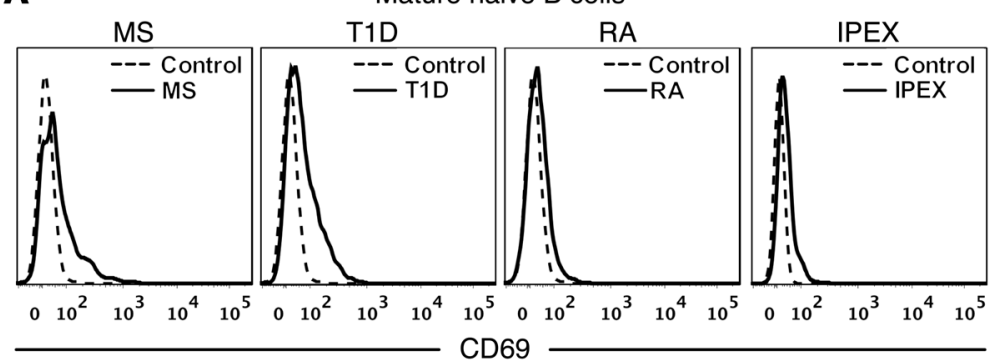

C New emigrant/transitional

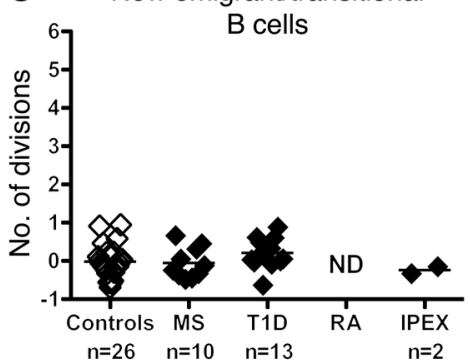

Mature naive $B$ cells

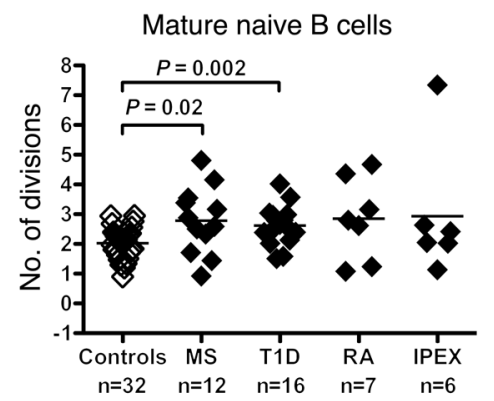

B

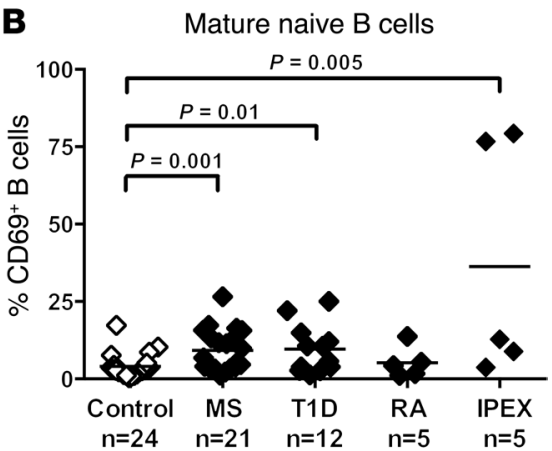

D

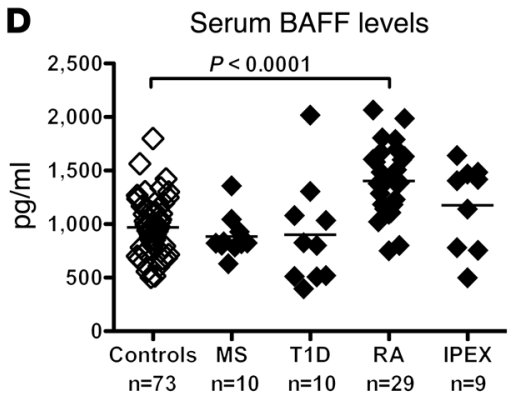

Figure 3

Mature naive B cells from patients with MS, T1D, and RA show an increased proliferative history. (A) Increased representative CD69 expression on CD19+CD27-CD10-CD21+ mature naive B cells and their percentages (B) in patients with MS, T1D, RA, and IPEX (bold lines) compared with healthy donors (dotted lines). (C) Evaluation of the number of cell divisions undergone in vivo by KREC analysis of new emigrant (left) and mature naive B cells (right) from healthy donors and MS, T1D, RA, and IPEX patients. Mature naive B cells from all patients showed increased proliferative history compared with controls. (D) Serum BAFF concentrations (pg/ml) in healthy donors and patients were measured by ELISA and found to be elevated only in RA patients. Statistical differences are indicated when significant.

abnormally enriched in $\mathrm{CD}^{2} 5 \mathrm{RO}^{+}$cells (Supplemental Figure 4 and ref. 12). Hence, MS and RA, but not T1D, patients harbor an increased proportion of memory Tregs, a feature potentially associated with an abnormal peripheral B cell tolerance checkpoint.

We report herein that a defective peripheral $B$ cell tolerance checkpoint is common to MS, T1D, and RA, whereas central B cell tolerance is only consistently impaired in T1D and RA. Hence, defects in the counterselection of developing autoreactive B cell clones in the bone marrow seem to be critical for the development of RA and T1D, but are dispensable for MS. Recent genome-wide association studies (GWAS) have demonstrated that several susceptibility alleles are shared among different autoimmune diseases (17). For example, the C1858T polymorphism in the PTPN22 gene is one of the highest-risk alleles for developing T1D and RA, but is not associated with MS. This PTPN22 variant results in blunting both $\mathrm{T}$ cell receptor (TCR) and B cell receptor (BCR) signaling, which likely regulates central tolerance $(18,19)$. In agreement with this hypothesis, we recently demonstrated that healthy donors carrying the C1858T PTPN22 risk allele are deficient in the removal of autoreactive $\mathrm{B}$ cells in the bone marrow (6). This finding is similar to that observed in T1D and RA patients, revealing that this susceptibility allele has a dominant effect and is sufficient to confer the impaired central $B$ cell tolerance associated with T1D and RA (5, 6). Many other susceptibility genes associated with T1D and RA, such as $B L K, L Y N$, PTPN2, and BANK1, also affect BCR signaling pathways (20) and may interfere with the removal of developing autoreactive $B$ cells. In contrast, none of these polymorphisms, including the C1858T PTPN22 variant, are associated with MS, further demonstrating that altered B cell intrinsic pathways controlling central B cell tolerance are not necessary for the development of this autoimmune disease. This notion is also supported by recent GWAS data indicating that the disease-associated polymorphisms in MS mainly affect $\mathrm{T}$ helper differentiation and APC-T cell interactions and not B cell intrinsic pathways (21). Our results are in agreement with these observations in that the maintenance of the peripheral B cell tolerance checkpoint that is specifically defective in MS patients seems to involve T-B cell interactions, requiring functional CD40L and MHC class II molecules (9). Importantly, peripheral tolerance defects similar to those in MS patients were identified in FOXP3-deficient IPEX patients whose Treg functions are impaired, demonstrating a critical role for Tregs in controlling this checkpoint (12). In addition, altered B cell features characteristic of nonfunctional Tregs were associated with MS as well as T1D and RA, further pointing to the possible role for Treg defects in these autoimmune conditions. The increased proportion of $\mathrm{CD}^{2} \mathrm{RO}^{+}$Tregs found in both MS and IPEX patients also supports this hypothesis. Moreover, a defective suppressive function linked to increased IFN- $\gamma$ production has previously been observed with memory Tregs from MS patients, further suggesting that abnormal Tregs in MS may be responsible for the impaired peripheral B cell tolerance checkpoint in these patients $(22,23)$.

In summary, our findings demonstrate subtle differences in B cell tolerance in different autoimmune diseases. Defects in the counterselection of autoreactive $\mathrm{B}$ cells in the bone marrow appear to be a dominant feature of RA and T1D, but not MS. In contrast, most patients with MS have a specific peripheral B cell tolerance checkpoint defect, potentially resulting from defective Treg function. Differences in 
early B cell tolerance checkpoint dysfunction between T1D, RA, and MS may have important consequences for the efficacy of anti-B cell therapy in these autoimmune diseases. Rituximab shows efficacy in delaying T1D and can improve clinical presentation in RA. However, the possibility remains that central B cell tolerance defects in these patients may allow post-rituximab production of a large population of autoreactive B cells in the bone marrow that could then lead to a rapid relapse in these patients after treatment termination $(7,8)$. In contrast, B cell-depletion therapy that eliminates autoreactive B cells in MS patients may result in more sustained remission (3) because newly generated $\mathrm{B}$ cells exiting the bone marrow will be properly counterselected in most patients.

\section{Methods}

Patients. Untreated MS patients with the relapsing-remitting form of the disease were recruited for the study (Supplemental Table 1). Healthy donors (Supplemental Table 1) as well as T1D and RA patients analyzed for antibody reactivity were previously reported (4-6, 9-11). Additional untreated patients with MS, T1D, or RA as well as age-matched healthy donors were recruited for the assessment of $\mathrm{B}$ cell and Treg phenotype, proliferation history, and BAFF serum concentrations (Figure 3 and Supplemental Figures 3 and 4).

Cell staining and sorting, cDNA, RT-PCR, antibody production, ELISAs, and indirect fluorescence assays. Single $\mathrm{CD} 21^{\mathrm{lo}} \mathrm{CD} 10^{++} \mathrm{IgM}{ }^{\mathrm{hi}} \mathrm{CD} 27^{-}$new emigrant/transitional and $\mathrm{CD} 21^{+} \mathrm{CD} 10^{-} \mathrm{IgM}^{+} \mathrm{CD} 27^{-}$mature naive $\mathrm{B}$ cells from patients and control donors were sorted on a FACSAria flow cytometer (BD) into 96-well PCR plates, and antibody reactivity was tested as previously described (4). See Supplemental Methods for details on the white matter extract ELISA. Serum BAFF concentrations were determined by ELISA according to the manufacturer's instructions (R\&D Systems).

Flow cytometry. The following antibodies were used for flow cytometric stainings: CD19-APC-Cy7, CD27-PerCP-Cy5.5, CD10-PE-Cy7, IgM-FITC, CD21-APC, CD69-PE, CD80-FITC, CD86-APC, CD4-APC-Cy7, CD25-PECy7, CD127-PerCP-Cy5.5, and CD45RO-Pacific Blue (all from BioLegend), and CD3-eFluor 605NC (eBioscience). Intracellular staining for FOXP3 Alexa Fluor 488 (clone PCH101; eBioscience), was performed using the Foxp3/Transcription Factor Staining Buffer Set (eBioscience) according to the manufacturer's instructions.

1. Nylander A, Hafler DA. Multiple sclerosis. J Clin Invest. 2012;122(4):1180-1188.

2. Büdingen von H-C, Bar-Or A, Zamvil SS. B cells in multiple sclerosis: connecting the dots. Curr Opin Immunol. 2011;23(6):713-720.

3. Hauser SL, et al. B-cell depletion with rituximab in relapsing-remitting multiple sclerosis. $N$ EnglJ Med. 2008;358(7):676-688.

4. Wardemann H, Yurasov S, Schaefer A, Young JW, Meffre E, Nussenzweig MC. Predominant autoantibody production by early human B-cell precursors. Science. 2003;301(5638):1374-1377.

5. Samuels J, Ng Y-S, Coupillaud C, Paget D, Meffre E. Impaired early B-cell tolerance in patients with rheumatoid arthritis. J Exp Med. 2005;201(10):1659-1667.

6. Menard L, et al. The PTPN22 allele encoding an R620W variant interferes with the removal of developing autoreactive B cells in humans. J Clin Invest. 2011;121(9):3635-3644.

7. Edwards JCW, et al. Efficacy of B-cell-targeted therapy with rituximab in patients with rheumatoid arthritis. N Engl J Med. 2004;350(25):2572-2581.

8. Pescovitz MD, et al. Rituximab, B-lymphocyte depletion, and preservation of beta-cell function. N Engl J Med. 2009;361(22):2143-2152.

9. Hervé $\mathrm{M}$, et al. CD40 ligand and MHC class II expression are essential for human peripheral 2008;29(5):746-757.
KREC assay. The ratio of KREC joints (signal joint) to JK-CK recombination genomic joints (coding joint) was determined as previously described (13). See Supplemental Methods for details.

Statistics. Differences between patients and healthy donors were analyzed for statistical significance with Mann-Whitney $U$ tests using GraphPad Prism software (GraphPad). $P$ values of less than 0.05 were considered statistically significant.

Study approval. All participants provided informed consent prior to participation in this study. All aspects of the study were approved by the Yale University School of Medicine Human Investigation Committee, New Haven, Connecticut, USA.

\section{Acknowledgments}

We thank S. Rudchenko, L. Devine, and C. Wang for cell sorting. This work was supported by grants from the NIH-NIAID (AI061093, AI071087, AI082713, and AI095848, to E. Meffre); the Sigrid Juselius Foundation, the Finnish Medical Foundation, and the Saastamoinen Foundation (to T. Kinnunen); the German Research Foundation (DFG, MO 2160/2-1, to H. Morbach); the Rubicon, Netherlands Organisation for Scientific Research (to T. Cantaert); and the Nancy Davis Foundation for Multiple Sclerosis (to K.C. O'Connor). D.A. Hafler received support from the National MS Society Collaborative Research Center Award (CA1061-A-18), from NIH grants (AI045757, AI046130, AI070352, AI039671), and from a Jacob Javits Merit Award (NS2427) from the National Institute of Neurological Disorders and Stroke and the Nancy Taylor Foundation for Chronic Diseases, Inc.

Received for publication January 11, 2013, and accepted in revised form March 21, 2013.

Address correspondence to: Eric Meffre, Yale University School of Medicine, 300 George Street, New Haven, Connecticut 06511, USA. Phone: 203.737.4535; Fax: 203.785.7903; E-mail: Eric.meffre@yale.edu.

Tuure Kinnunen's present address is: Department of Clinical Microbiology, University of Eastern Finland, Kuopio, Finland.
B-cell tolerance. J Exp Med. 2007;204(7):1583-1593.

10. Isnardi I, et al. IRAK-4- and MyD88-dependent pathways are essential for the removal of developing autoreactive B cells in humans. Immunity.

11. Sauer AV, Morbach H, Brigida I, Ng Y-S, Aiuti A, Meffre E. Defective B-cell tolerance in adenosine deaminase deficiency is corrected by gene therapy. J Clin Invest. 2012;122(6):2141-2152.

12. Kinnunen T, et al. Accumulation of peripheral autoreactive $B$ cells in the absence of functional human regulatory T cells. Blood. 2013;121(9):1595-1603.

13. van Zelm MC, Szczepanski T, van der Burg M, van Dongen JJM. Replication history of B lymphocytes reveals homeostatic proliferation and extensive antigen-induced B-cell expansion. J Exp Med. 2007;204(3):645-655.

14. Mackay F, Schneider P. Cracking the BAFF code. Nat Rev Immunol. 2009;9(7):491-502.

15. Haas J, et al. Prevalence of newly generated naive regulatory $\mathrm{T}$ cells (Treg) is critical for Treg suppressive function and determines Treg dysfunction in multiple sclerosis. J Immunol. 2007;179(2):1322-1330.

16. Venken K, Hellings N, Broekmans T, Hensen K, Rummens J-L, Stinissen P. Natural naive $\mathrm{CD} 4{ }^{+} \mathrm{C}$ $\mathrm{D} 25^{+} \mathrm{CD} 127$ low regulatory $\mathrm{T}$ cell (Treg) development and function are disturbed in multi- ple sclerosis patients: recovery of memory Treg homeostasis during disease progression. JImmunol. 2008;180(9):6411-6420.

17. Cotsapas $C$, et al. Pervasive sharing of genetic effects in autoimmune disease. PLoS Genet. 2011;7(8):e1002254.

18. Rieck M, Arechiga A, Onengut-Gumuscu S, Greenbaum C, Concannon P, Buckner JH. Genetic variation in PTPN22 corresponds to altered function of $\mathrm{T}$ and B lymphocytes. J Immunol. 2007;179(7):4704-4710.

19. Arechiga AF, et al. Cutting edge: the PTPN22 allelic variant associated with autoimmunity impairs B cell signaling. J Immunol. 2009;182(6):3343-3347.

20. Cho JH, Gregersen PK. Genomics and the multifactorial nature of human autoimmune disease. NEngl J Med. 2011;365(17):1612-1623.

21. Sawcer $S$, et al. Genetic risk and a primary role for cell-mediated immune mechanisms in multiple sclerosis. Nature. 2011;476(7359):214-219.

22. Viglietta V, Baecher-Allan C, Weiner HL, Hafler DA. Loss of functional suppression by $\mathrm{CD} 4{ }^{+} \mathrm{CD} 25^{+}$regulatory $\mathrm{T}$ cells in patients with multiple sclerosis. J Exp Med. 2004;199(7):971-979.

23. Dominguez-Villar M, Baecher-Allan CM, Hafler DA. Identification of T helper type 1-like, Foxp3(+) regulatory $\mathrm{T}$ cells in human autoimmune disease. Nat Med. 2011;17(6):673-675. 